\title{
Hybrid approach to the comprehensive stage II operation in a subset of single-ventricle variants
}

\author{
William M. DeCampli, MD, PhD, Craig E. Fleishman, MD, and David G. Nykanen, MD
}

\section{ABSTRACT}

Objective: The objective of a hybrid approach to staged palliation of singleventricle anomalies is designed to minimize the trauma of the first stage. However, the second stage is a complex procedure that may negate the advantages of the first stage. We sought to devise a "hybrid" approach to the second stage when aortic outflow is expected to remain unobstructed.

Methods: The procedure involves a simple incision into the main pulmonary artery, dilation/stenting of the ductal continuation, formation of a stented baffle between the branch pulmonary arteries' orifices, and a bidirectional Glenn connection. It avoids dissection of the distal arch and ductal continuation and obviates the need for a Damus-Kaye-Stansel connection. We carried out this procedure in 2 patients, one with unbalanced atrioventricular canal and the other with mitral atresia.

Results: Both patients underwent an uncomplicated operative procedure. Both patients were successfully weaned from the ventilator, with no clinically evident neurologic injury. The first patient died of complications related to thrombosis of the left pulmonary artery before initiation of anticoagulation. The second patient is alive and well 1 year postoperation with no obstruction to either systemic or pulmonary flow and no baffle leak and good right ventricle function.

Conclusions: This hybrid comprehensive stage II operation appears feasible and technically simpler than the conventional comprehensive stage II procedure. It is applicable to a subset of single-ventricle cases in which aortic outflow is anticipated to remain unobstructed. We recommend early postoperative anticoagulation to avoid early left pulmonary artery thrombosis. (J Thorac Cardiovasc Surg 2015;149:1095-100)

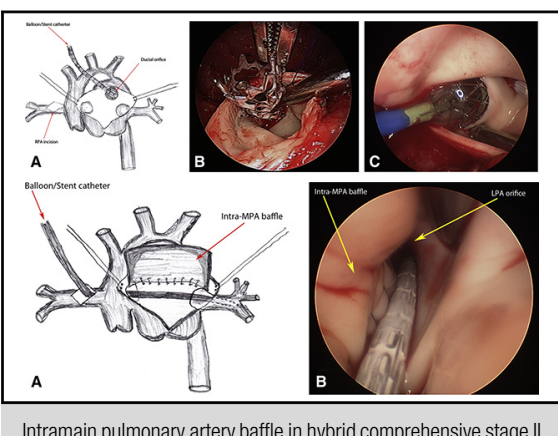

Intramain pulmonary artery baffle in hybrid comprehensive stage II procedure.

\section{Central Message}

This hybrid comprehensive stage II operation is applicable to a subset of single-ventricle cases in which aortic outflow is anticipated to remain unobstructed. It appears feasible and technically simpler than the conventional comprehensive stage II procedure. We recommend early postoperative anticoagulation to avoid early left pulmonary artery thrombosis.

\section{Perspective}

The objective of a hybrid approach to staged palliation of single-ventricle anomalies is to minimize the trauma of the first stage. However, the second stage is a complex procedure with potential complications that may negate the advantages of the first stage. We present a hybrid approach to the second stage in a subset of patients with sufficient antegrade aortic outflow to sustain upper body perfusion. The technique is less complicated than the conventional comprehensive stage II operation, avoiding distal arch dissection and reconstruction and an aortopulmonary anastomosi and with further technical developments may be achieved without hypothermia or cardiac and circulatory arrest.

See Editorial Commentary page 1101
The "hybrid Norwood" sequence of staged palliation is a potential alternative to the conventional surgical management of patients with hypoplastic left heart syndrome (HLHS) and single-ventricle variants. The procedure consists of bilateral branch pulmonary artery banding, ductal stenting, and, when indicated, balloon atrial septostomy.

\footnotetext{
From The Heart Center at Arnold Palmer Hospital for Children, Orlando, Fla; and College of Medicine, University of Central Florida, Orlando, Fla.

This work was supported by the Orlando Health Foundation (grant \#OHF10512).

Received for publication Sept 8, 2014; revisions received Nov 21, 2014; accepted for publication Nov 29, 2014; available ahead of print Jan 13, 2015.

Address for reprints: William M. DeCampli, MD, PhD, Division of Cardiothoracic

Surgery, The Heart Center at Arnold Palmer Hospital for Children, 92 W Miller

St, Orlando, FL 32806 (E-mail: William.decampli@ orlandohealth.com).

$0022-5223 / \$ 36.00$

Copyright (c) 2015 by The American Association for Thoracic Surgery

http://dx.doi.org/10.1016/j.jtcvs.2014.11.081
}

The procedure is typically performed without cardiopulmonary bypass. The putative advantage of this approach is the avoidance of major surgical trauma in the newborn period. Although some centers report laudable outcomes for the hybrid approach, others report intermediate-term outcome (mortality) no better than that of the conventional staged palliation. ${ }^{1-5}$ One study found no difference in neurodevelopmental outcome at 1 year between patients managed with the conventional approach and those managed with the hybrid approach.

Although the first stage of the hybrid approach avoids a prolonged operation with cardiopulmonary bypass, deep hypothermia, and circulatory arrest, these techniques are typically required at the time of the "comprehensive stage II" operation. Additionally, this operation requires management of the ductal stent and, in some cases, bilateral 


$$
\begin{aligned}
& \text { Abbreviations and Acronyms } \\
& \begin{aligned}
\text { AV } & =\text { atrioventricular } \\
\text { DILV } & =\text { double-inlet left ventricle } \\
\text { DKS } & =\text { Damus-Kaye-Stansel } \\
\text { HLHS } & =\text { hypoplastic left heart syndrome } \\
\text { LPA } & =\text { left pulmonary artery } \\
\text { LV } & =\text { left ventricle } \\
\text { MPA } & =\text { main pulmonary artery } \\
\text { PA } & =\text { pulmonary artery } \\
\text { PDA } & =\text { patent ductus arteriosus } \\
\text { PG } & =\text { Palmaz Genesis } \\
R & =\text { right } \\
\text { RPA } & =\text { right pulmonary artery } \\
\text { RV } & =\text { right ventricle } \\
S & =\text { superior } \\
\text { TA } & =\text { tricuspid atresia } \\
\text { VA } & =\text { ventricular-arterial }
\end{aligned}
\end{aligned}
$$

pulmonary artery reconstruction because of band-induced trauma. The learning curve is known to be steep. ${ }^{5}$ It remains a leap of faith that this deferral of trauma will result in better long-term survival and neurodevelopment for the patient.

To further reduce the total trauma to the patient, we propose an alternative technique to the standard comprehensive stage II operation, applicable to the subset of patients with sufficient antegrade aortic flow to sustain upper body perfusion. The technique avoids the creation of a Damus-KayeStansel (DKS) connection and surgical arch reconstruction entirely and, with further technical development, may be achieved without hypothermia, cardiac and circulatory arrest.

\section{PATIENTS}

\section{Case 1}

The patient was a full-term 3.3-kg newborn with Down syndrome. Echocardiography demonstrated an unbalanced complete atrioventricular (AV) canal defect. The left ventricular (LV) volume index was $14 \mathrm{~mL} / \mathrm{m}^{2}$. The $\mathrm{LV} /$ right ventricle (RV) effective valve area ratio was 0.42 (AV valve index 0.29). The LV outflow track was not obstructed, but the aortic arch was hypoplastic, with a midtransverse arch-to-ascending aortic diameter ratio of 0.34 . The isthmus was also hypoplastic and there was a large ductal arch. On day 4, the neonate underwent the hybrid stage I procedure. Following hospital discharge, echocardiography demonstrated that the ductal stent had migrated somewhat into the descending aorta, but the ductus remained patent with a gradient less than $10 \mathrm{~mm} \mathrm{Hg}$. At 5 months $(7 \mathrm{~kg})$, catheterization demonstrated unobstructed antegrade aortic flow to the arch vessels but no appreciable flow from the ascending aorta into the descending aorta. The aortic annulus, aortic root, ascending aorta, and transverse arch diameters were $8 \mathrm{~mm}(\mathrm{z}=-0.9), 14 \mathrm{~mm}(\mathrm{z}=+1.7), 12 \mathrm{~mm}$ $(\mathrm{z}=+1.2)$, and $6 \mathrm{~mm}(\mathrm{z}=-1.9)$, respectively. The mean systolic gradient through the ductus into the descending aorta was $12 \mathrm{~mm} \mathrm{Hg}$. Mean distal left and right pulmonary artery (LPA and RPA, respectively) pressures were 24 and $10 \mathrm{~mm} \mathrm{Hg}$, respectively. The patient underwent the following hybrid stage II procedure:

\section{Technique}

Through a median sternotomy, we mobilized the heart, great vessels, and innominate and superior caval veins and divided the azygous vein. We did not mobilize the distal arch, aortic isthmus, or descending aorta. We placed dual arterial cannulas in the innominate artery and pulmonary artery (PA) trunk and venous cannulas in the right atrium and (later) innominate vein, then commenced cardiopulmonary bypass. After cooling to $18^{\circ} \mathrm{C}$ degrees, we clamped the ascending aorta, arrested the heart, and then removed the polytetrafluoroethylene bands from the branch pulmonary arteries. We then occluded the arch branches, removed the PA cannula, and commenced antegrade regional cerebral perfusion. (Occlusion of the arch branches may not be necessary if the distal arch is already discontinuous or if one can dilate or re-stent the ductal arch without a bloodfree descending aorta. In this case, aortic, rather than innominate artery, cannulation can be performed, with perfusion to the entire upper body.) We did not occlude
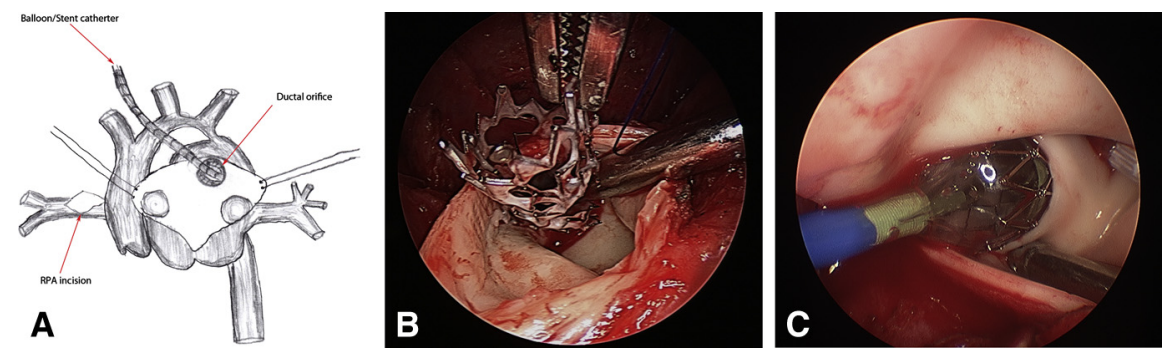

FIGURE 1. After commencing cardiopulmonary bypass with 1 or 2 arterial cannulas and a right atrium cannula, the branch pulmonary arteries are debanded. A, Under selective cerebral perfusion, an incision is made into the RPA at the proposed site of superior vena cava connection, usually at or just distal to the site of prior banding. Then, a longitudinal incision is made into the large main pulmonary artery. The location of the branch pulmonary artery and ductal orifices are noted. B, A protruding end of the original ductal stent is trimmed back. C, The ductal stent is then dilated under direct vision, and a second stent placed as needed and position determined by videoscope. RPA, Right pulmonary artery. 

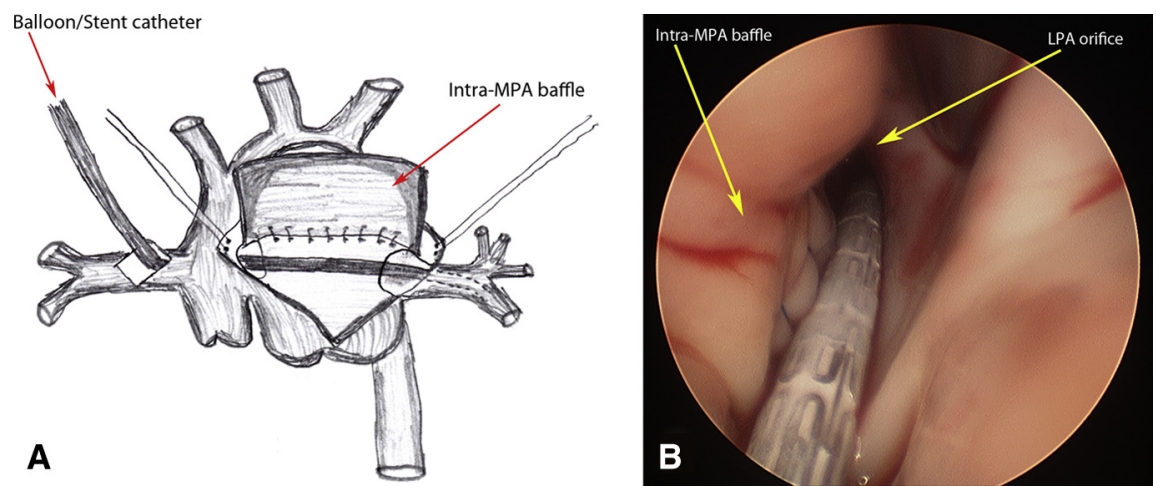

FIGURE 2. A, A guide wire and pulmonary artery stent catheter are advanced through the right pulmonary artery incision into the left lower lobe artery using videoscopic guidance. B, A pulmonary artery homograft baffle is partially sewn in place from the left to the right pulmonary artery orifice (intra-MPA baffle). The intimal side of the graft is oriented to lie within the pulmonary pathway because the flow there is of relatively low velocity and less pulsatile than that of the systemic pathway. MPA, Main pulmonary artery; $L P A$, left pulmonary artery.

the descending aorta. We then opened the RPA at the proposed site of superior vena cava anastomosis. After making a longitudinal incision into the pulmonary trunk, we deployed a single Palmaz Genesis (PG) 2510B stent (Cordis, Bridgewater, NJ) over a 9-2 Opta Pro balloon (Cordis) under direct vision across the ductal orifice (Figure 1). We then advanced a guide wire from the RPA incision leftward into the left lower lobe artery. Next we partially sutured a baffle within the pulmonary bifurcation from the right to the LPA orifices using pulmonary homograft material (Figure 2).
Then, using the guidance of a 5-mm videoscope, we advanced an iCast $22 \mathrm{~mm} \times 5 \mathrm{~mm}$ covered stent (Atrium Medical Corporation, Hudson, $\mathrm{NH}$ ) over an $8 \mathrm{~mm} \times 2 \mathrm{~cm}$ Opta Pro balloon (Cordis) through the right pulmonary arteriotomy into the pulmonary bifurcation then into the orifice of the LPA, positioning the stent end just proximal to the lobar branching. We then deployed the stent, fixing it in place using a 6-0 Prolene (polypropylene; Ethicon, Inc, Somerville, NJ) stitch through the anterior wall of each branch PA orifice to avoid stent migration (Figure 3, A).
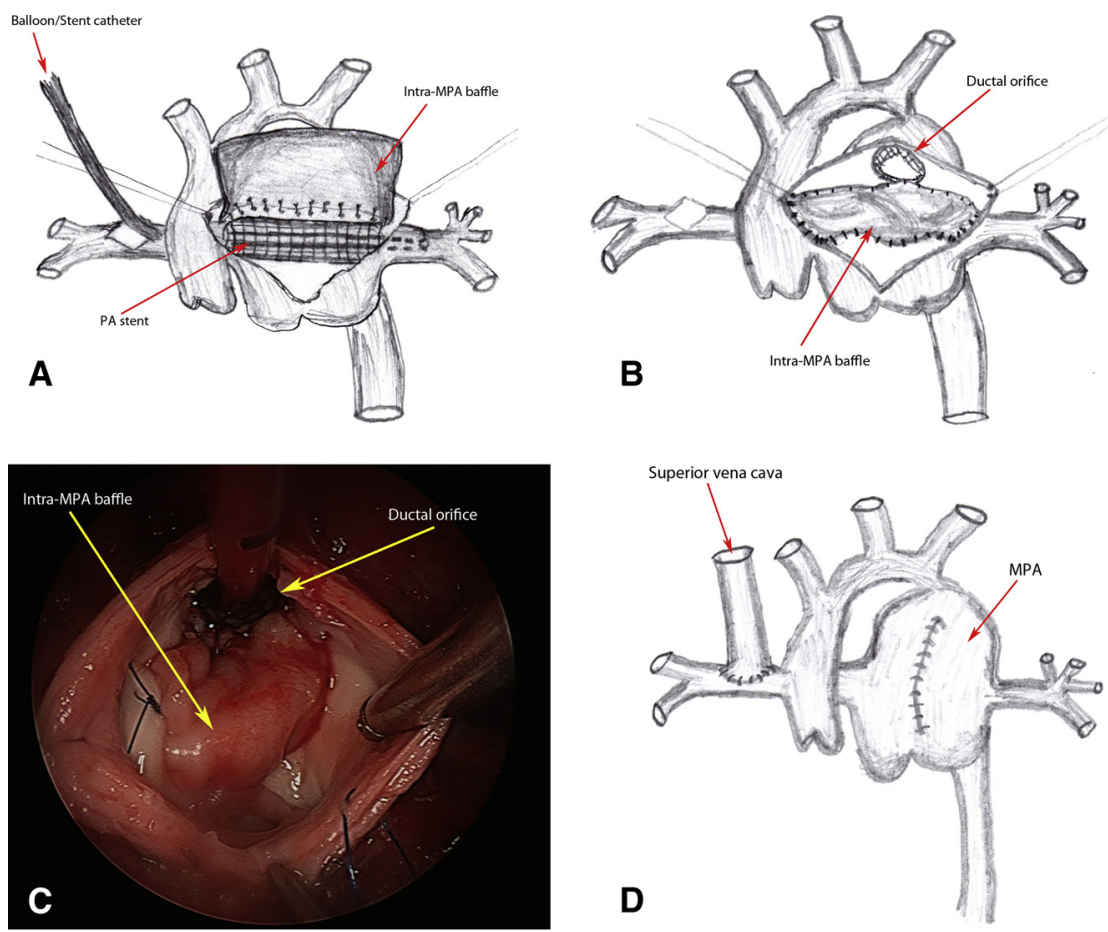

FIGURE 3. A, The PA stent is first deployed. It should extend into each PA orifice by about $5 \mathrm{~mm}$. B, C, The baffle suture line is completed, coming up over each PA orifice and back down to the floor of the PA bifurcation. The baffle is trimmed to fit snugly around the stent. The stent is anchored near each PA orifice using a 5-0 Prolene (polypropylene; Ethicon) stitch to prevent migration. D, The incision in the PA is closed and the heart and vasculature de-aired. The PA arterial cannula is replaced and reperfusion and warming begun. A superior vena cava cannula is placed and the cavo-pulmonary anastomosis is then performed with homograft augmentation as needed. MPA, Main pulmonary artery; $P A$, pulmonary artery. 

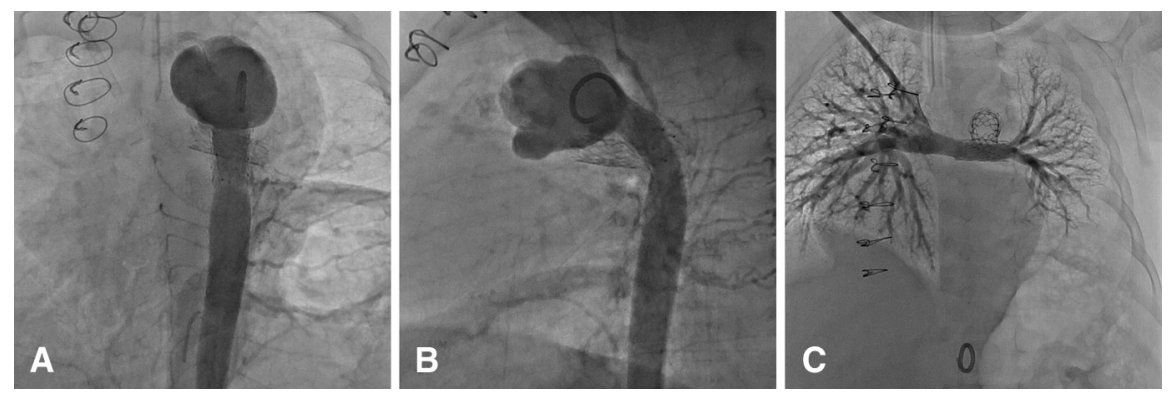

FIGURE 4. An "exit angiogram" is performed to confirm bilateral pulmonary artery flow without pulmonary or systemic obstruction and no baffle leak (left to right shunt). Anteroposterior (A) and lateral (B) views of pulmonary root injection, showing no systemic obstruction or shunt into the branch pulmonary arteries. C, Cavo-pulmonary injection showing unobstructed baffle and left pulmonary artery flow and absence of wash-in from the systemic circulation.

Stenting the baffle from PA orifice to orifice is required in order to prevent the baffle from being "crushed" by the outside systemic flow. Although suture fixation may not be necessary, we recommend it as there are considerable stresses exerted externally on the stent by the surrounding systemic circulation. We found it straightforward to deploy a stent in this position that can later be dilated to 15 to $18 \mathrm{~mm}$ - essentially adult size. We then completed the intra-arterial baffle (Figure 3, $B$ and $C$ ). The posterior part of the circumference of this baffle consisted of native PA bifurcation tissue to allow growth. Finally, we closed the pulmonary trunk incision; deaired the heart, pulmonary trunk, and aortic arch; replaced the PA cannula; removed all vascular clamps; and then recommenced full-flow cardiopulmonary bypass. While warming, we completed the cavo-pulmonary anastomosis (Figure 3, $D)$. Post-bypass echocardiography and "exit angiography" of the pulmonary circuit showed unobstructed flow into both lungs and no evidence of a systemic-to-pulmonary shunt (Figure 4). The systolic gradient between the right radial artery and femoral artery was 0 to $5 \mathrm{~mm} \mathrm{Hg}$. Lactate levels remained below $2.0 \mu \mathrm{g} / \mathrm{dL}$. Bleeding was easily controlled and the chest was closed.

\section{Clinical Course}

The patient made an initially unremarkable recovery and was weaned from the ventilator. On postoperative day 5 , she became hypoxemic and chest radiograph showed opacification of the left hemithorax. No flow was seen in the LPA by echocardiography and computed tomographic angiography. The patient was heparinized and direct angiography confirmed extensive thrombus in the intra-pulmonary covered stent and LPA and its lobar branches. Selective catheter infusion of tissue plasminogen activator was carried out and the patient continued on heparin. The patient required veno-venous extracorporeal membrane oxygenation postcatheterization and was weaned from support on postoperative day 22. Repeat angiography revealed no resolution of stent/LPA thrombosis. She remained on maximal ventilator support and succumbed to hypoxia on postoperative day 26.

\section{Case 2}

The patient was a 3.3-kg newborn infant with mitral atresia and double outlet right ventricle. Echocardiography showed a left-posterior aortic root, some crowding in the subaortic area but with no gradient, and unobstructed pulmonary outlet. The aortic annulus diameter was $5.3 \mathrm{~mm}$ $(\mathrm{z}=-2.3)$, ascending aorta $5.7 \mathrm{~mm}(\mathrm{z}=-1.8)$, the distal transverse arch $3.2 \mathrm{~mm}(\mathrm{z}=-3.1)$, and the isthmus 2.0 $\mathrm{mm}(\mathrm{z}=-3.5)$. The neonate underwent the hybrid stage 1 procedure on day 6 . The patient had a number of hospital readmissions for recurrent pulmonary infections during the inter-stage. At age 5 months and $6.3 \mathrm{~kg}$, catheterization showed good RV function and no gradient from either the $\mathrm{RV}$ to the descending aorta or from the RV to the left subclavian artery. The aortic annulus and ascending aorta had grown commensurate with somatic growth. The mean PA pressure was $11 \mathrm{~mm} \mathrm{Hg}$ and the RV end-diastolic pressure was $8 \mathrm{~mm} \mathrm{Hg}$. With the history of pulmonary infections, the patient was determined to be a relatively high risk for the conventional comprehensive stage II operation and therefore underwent the hybrid comprehensive stage II operation described below.

\section{Technique}

Preparation and perfusion technique proceeded as described above. After commencing regional cerebral perfusion, we opened the main PA longitudinally and trimmed the ductal stent back to flush with the PA bifurcation. We then stented the ductal-aortic continuation with a PG 1910B stent over an 8-2 Opta balloon. Within the PA bifurcation, we partially sutured a wide, thickwalled pulmonary homograft baffle between the left and right PA orifices. We then made an incision into the mid-right PA. Through this, we advanced a PG 1910B bare metal stent over an 8-2 Opta balloon so it straddled the PA orifices, using a 5-mm videoscope to ensure that the left lobar PA branches were not compromised. We deployed the stent and anchored it in place to the bifurcation tissue as described above. We then completed the intra-PA baffle. The remainder of the case proceeded as in case 1 . 


\section{Clinical Course}

The patient was extubated on day 3 and managed postoperatively with heparin then transitioned to enoxaparin. A parainfluenza infection was associated with prolonged pulmonary compromise, but she recovered and was discharged on postoperative day 53 on enoxaparin injections. Aspirin was substituted for enoxaparin at 3 months postoperation. At age 11 months, she underwent balloon dilation of both the ductal stent and stent. Angiography revealed no shunt or aneurysm formation of the intrapulmonary baffle. Echocardiography during clinical follow-up at age 15 months and $9.7 \mathrm{~kg}$ showed good RV function, unobstructed cavopulmonary connection, unobstructed flow from the right ventricle to the transverse arch, unobstructed flow out the native aortic root, peak Doppler gradient of $25 \mathrm{~mm} \mathrm{Hg}$ across the ductal-to-descending aorta stents, and no intrapulmonary systemic baffle-to-PA shunt (Figure 5).

\section{DISCUSSION}

The principal motivation for the hybrid approach to staged palliation of HLHS and other single-ventricle variants is to avoid "trauma" to these patients in the neonatal period during the first-stage procedure. On the other hand, trauma is increased at the second stage. In a recent report by Schranz et al of 107 patients undergoing the hybrid staged approach, the comprehensive stage II mortality was $9 \%$, comparable to that of the Norwood procedure in experienced institutions. ${ }^{7}$ It is not yet known whether this strategy confers a net advantage to the patient. A substantial decrease in trauma at the second stage, at least in a subset of anatomic variants, could tip the balance in favor of the hybrid approach.

The present report exemplifies the principles of a technique that could achieve this objective in a subset of single-ventricle patients who have unobstructed flow through the subaortic outflow track and aortic root. The essence of the technique is to (1) maintain upper body perfusion through the native aortic root and arch, (2) maintain lower body perfusion through the ductal vestige, and
(3) isolate the pulmonary circulation using a stented intrapulmonary bifurcation baffle. The procedure completely avoids arch reconstruction and creation of a Damus connection. In principle, it can be performed through a limited PA incision, short regional cerebral perfusion duration, and little bleeding. Lower body circulatory arrest could be limited to a few minutes by stenting the ductus under fluoroscopy and by placing an occlusive cannula into the ductal continuation after opening the PA bifurcation. In the infrequent case where the ductal continuation could not be rendered unobstructed by stenting, the pulmonary trunk incision could be extended through the ductus and slightly onto the descending aorta, then patched. Should a suitable "covered stent" become available, the procedure could be further simplified by fluoroscopic placement of the pulmonary bifurcation stent. In that case, the entire procedure could be completed on warm cardiopulmonary bypass and beating heart with no incision into a systemic vessel.

The principal criterion for application of this technique is evidence that the arch vessels will continue to be adequately perfused via the native subaortic region and aortic root. Anatomic variants where this is sometimes (but not invariably) the case include HLHS with mitral and aortic stenosis, mitral atresia with ventricular septal defect, hypoplastic ventricle with interrupted aortic arch, double-inlet left ventricle (DILV), tricuspid atresia with ventricular-arterial discordance (TA/VA discordance), unbalanced atrioventricular canal, and certain forms of complex heterotaxy.

The patient in case 1 sustained early thrombosis of the intrapulmonary covered stent and LPA, which ultimately proved fatal. LPA thrombosis following single-ventricle palliation is well described. ${ }^{8-10}$ We speculate that delayed heparinization may have led to early thrombosis, and we now recommend use of a noncovered stent and early anticoagulation.

This procedure has several potential advantages, as follows: (1) limited arterial incision and baffle construction, shortening the duration of hypothermia, and regional cerebral
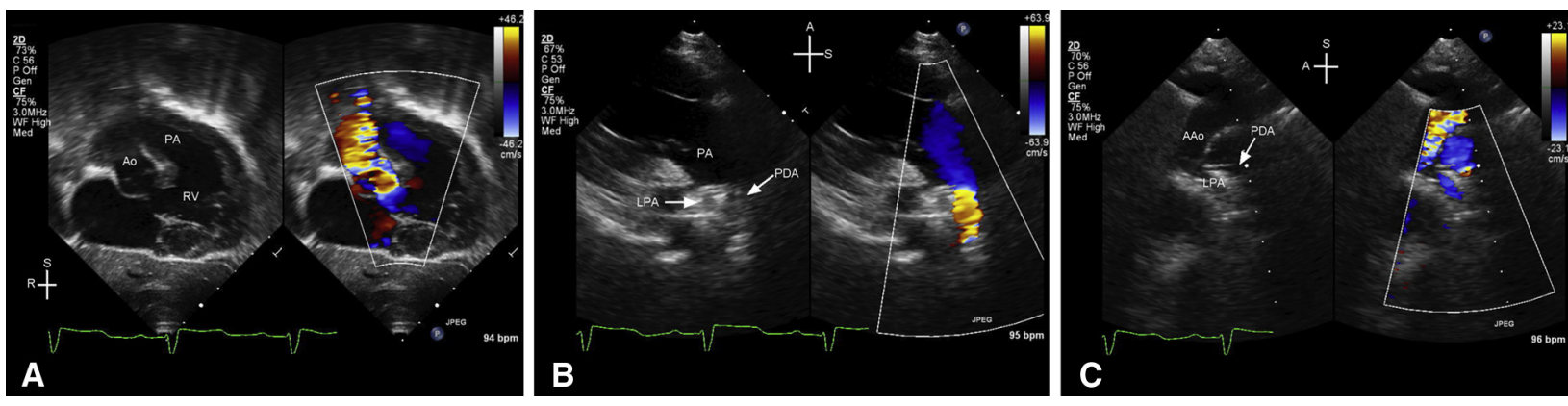

FIGURE 5. Echocardiographic images shown for case 2 at 10 months postoperation, age 15 months. A, Subcostal view shows that the anteriorly deviated conal septum crowds the subaortic area somewhat but is not obstructive to "upper body" (native aortic) flow. B, Parasternal view shows anatomic relationship between the main PA and the intra-PA baffle and LPA. Color Doppler shows flow from PA into stented PDA with no shunt (ie, baffle leak) into the LPA. $\mathrm{C}$, Suprasternal view shows separate AAo, PDA, and LPA flows. Ao, Aorta root; $P A$, pulmonary artery; $R V$, right ventricle; $R$, right; $S$, superior; $L P A$, left pulmonary artery; $P D A$, patent ductus arteriosus; $A A o$, ascending aorta. 
perfusion; (2) with the usage of a suitable intrapulmonary bifurcation stent, complete elimination of hypothermia, cardiac arrest, and an interval of regional perfusion; and (3) complete avoidance of mobilization and reconstruction of the arch, isthmus, and descending aorta, decreasing the risk of substantial bleeding, patch distortion with subsequent arch obstruction and recurrent nerve and phrenic nerve injury.

The procedure has several potential disadvantages, including (1) late development of inadequate aortic outflow track (subaortic, valvar, or supravalvar), (2) LPA stenosis, (3) stent migration with subsequent LPA occlusion, (4) baffle leak (aortopulmonary shunt), and (5) recurrent ductal arch stenosis. In most cases, complications are avoidable or treatable by careful patient selection, selection of appropriately sized stents dilatable to achieve "adult" flow requirements, and by aggressive follow-up and catheter-based reintervention.

Although the proposed procedure requires placement of 1 or 2 stents, stent management with the conventional and standard hybrid staged approaches is common. Dave et al reported that the incidence of branch PA stenting was $17 \%$ during conventional management and $38 \%$ after the hybrid stage I or II. ${ }^{11}$ Reinhardt et al showed that, in the conventional staged approach, $37 \%$ of patients ultimately required catheter reintervention. ${ }^{12}$ Although the ability of stented vessels to sustain the circulation into adulthood is a justifiable concern, multiple studies have shown that aortic and PA stents placed even in infancy can be dilated to an adult diameter with an average of 3 interventions. ${ }^{13-15}$

Whereas in most cases the initial assessment of aortic outflow adequacy is predictive of future adequacy, in others it may remain uncertain, such as those with bulboventricular foramen (DILV or TA/VA discordance). In this particular subset, studies have shown that the initial size of the foramen must be quite large $(\mathrm{z}>+2.5$ relative to the normal aortic outflow diameter) to ensure absence of obstruction over time. ${ }^{16}$ On the other hand, the proposed technique requires only that the aortic outflow provide $30 \%$ to $40 \%$ of the cardiac output ("upper body flow"), so the criteria for an adequate aortic outflow may be liberalized (though to what degree remains uncertain). Should this complication develop after the proposed operation, one may mitigate it by performing a side-to-side aortopulmonary connection with patch (a forme fruste of DKS), or by directly enlarging the foramen/outflow tract.

In this initial 2-case report, we obviously could not demonstrate the superiority of the proposed procedure over the usual comprehensive stage II operation. Our limited experience, however, is that the proposed procedure is significantly less surgically complicated than the conventional operation as it avoids distal arch dissection and reconstruction. For most cases in which this new technique is applied, the final palliative stage (Fontan procedure) would be conducted in the usual manner except for the need for dual arterial cannulation for cardiopulmonary bypass.
When the hybrid approach is used as a bridge to 2-ventricle repair, takedown of the proposed stage II reconstruction should be straightforward. ${ }^{17}$ We suggest that performance of the hybrid comprehensive stage II operation should be considered in selected patients undergoing the hybrid Norwood approach to single-ventricle palliation.

\section{Conflict of Interest Statement}

Authors have nothing to disclose with regard to commercial support.

\section{References}

1. Brescia AA, Jureidini S, Danon S, Armbrecht E, Fiore AC, Huddleston CB Hybrid versus Norwood procedure for hypoplastic left heart syndrome: contemporary series from a single center. J Thorac Cardiovasc Surg. 2014;147:1777-82.

2. Knirsch W, Bertholdt S, Stoffel G, Stiasny B, Weber R, Dave H, et al. Clinical course and interstage monitoring after the Norwood and hybrid procedures for hypoplastic left heart syndrome. Pediatr Cardiol. 2014;35:851-6.

3. Lloyd DF, Cutler L, Tibby SM, Vimalesvaran S, Qureshi SA, Rosenthal E, et al. Analysis of preoperative condition and interstage mortality in Norwood and hybrid procedures for hypoplastic left heart syndrome using the Aristotle scoring system. Heart. 2014;100:775-80.

4. Photiadis J, Sinzobahamvya N, Hraska V, Asfour B. Does bilateral pulmonary banding in comparison to Norwood procedure improve outcome in neonates with hypoplastic left heart syndrome beyond second-stage palliation? A review of the current literature. Thorac Cardiovasc Surg. 2012;60:181-8.

5. Galantowicz M, Cheatham JP, Phillips A, Cua CL, Hoffman TM, Hill SL, et al. Hybrid approach for hypoplastic left heart syndrome: intermediate results after the learning curve. Ann Thorac Surg. 2008;85:2063-70.

6. Knirsch W, Liamlahi R, Hug MI, Hoop R, von Rhein M, Pretre R, et al. Mortality and neurodevelopmental outcome at 1 year of age comparing hybrid and Norwood procedures. Eur J Cardiothorac Surg. 2012;42:33-9.

7. Schranz D, Bauer A, Reich B, Steinbrenner B, Recla S, Schmidt D, et al. Fifteenyear single center experience with the "Giessen hybrid" approach for hypoplastic left heart and variants: current strategies and outcomes. Pediatr Cardiol. 2014 [Epub ahead of print].

8. Hirono K, Ibuki K, Tomita H. Percutaneous catheter aspiration thrombectomy for the occluded stents of pulmonary artery in children with single ventricle physiology after Fontan surgery. Catheter Cardiovasc Interv. 2014;84:1153-6.

9. Zampi JD, Hirsch-Romano JC, Armstrong AK. Early cyanosis after stage II palliation for single ventricle physiology: etiologies and outcomes. World J Pediatr Congenit Heart Surg. 2013;4:367-72.

10. Manlhiot C, Brandão LR, Kwok J, Kegel S, Menjak IB, Carew CL, et al. Thrombotic complications and thromboprophylaxis across all three stages of single ventricle heart palliation. J Pediatr. 2012;161:513-9.

11. Dave H, Rosser B, Knirsch W, Hubler M, Pretre R, Kretschmar O. Hybrid approach for hypoplastic left heart syndrome and its variants: the fate of the pulmonary arteries. Eur J Cardiothorac Surg. 2014;46:14-9.

12. Reinhardt Z, De Giovanni J, Stickley J, Bhole VK, Anderson B, Murtuza B, et al. Catheter interventions in the staged management of hypoplastic left heart syndrome. Cardiol Young. 2014;24:212-9.

13. Kutty S, Burke RP, Hannan RL, Zahn EM. Hybrid aortic reconstruction for treatment of recurrent aortic obstruction after stage 1 single ventricle palliation: medium term outcomes and results of redilation. Catheter Cardiovasc Interv. 2011; 78:93-100.

14. Stanfill R, Nykanen DG, Osorio S, Whalen R, Burke RP, Zahn EM. Stent implantation is effective treatment of vascular stenosis in young infants with congenital heart disease: acute implantation and long-term follow-up results. Catheter Cardiovasc Interv. 2008;71:831-41.

15. Duke C, Rosenthal E, Qureshi SA. The efficacy and safety of stent redilatation in congenital heart disease. Heart. 2003;89:905-12.

16. Matitiau A, Geva T, Colan S, Sluysmans T, Parness I, Spevak P, et al. Bulboventricular foramen size in infants with double-inlet left ventricle or tricuspid atresia with transposed great arteries: influence on initial palliative operation and rate of growth. J Am Coll Cardiol. 1992;19:142-8.

17. Ballard G, Tibby S, Miller O, Krasemann T, Rosenthal E, Anderson D, et al. Growth of left heart structures following the hybrid procedure for borderline hypoplastic left heart. Eur J Echocardiogr. 2010;11:870-4. 